\title{
Comparison of centralized and distributed photovoltaic system power intermittency based on measured data at the municipal scale
}

\author{
Bryan E. Ellis ${ }^{1}$, Nathaniel Pearre ${ }^{1}$, Lukas Swan ${ }^{1}$ \\ ${ }^{1}$ Department of Mechanical Engineering, Dalhousie University, Halifax, Canada
}

\begin{abstract}
The decrease in solar photovoltaic (PV) system costs and regulatory barriers coupled with increased financial incentives and climate awareness has led to rapid deployment in both commercial and residential sectors. As solar is an intermittent resource, PV systems can negatively impact electricity grid stability due to increased power ramp-rates and temporal misalignment between energy production and load. Distributed systems, especially rooftop mounted residential, present a range of geometric alignments and spatial separation that when aggregated introduce temporal diversity. If these features reduce barriers to integration with the electricity grid, then they should be weighed against increased costs per rated power compared to larger commercial systems. To aid in such assessments, we compare power production data from 44 diverse residential PV systems spread across a large municipality $\left(1200 \mathrm{~km}^{2}\right)$, and one large rooftop commercial installation $(660 \mathrm{~kW})$ within the same area. These production data were used to calculate 5 and 15 -minute ramp rates and were contrasted against provincial load. The aggregated residential PV systems ramp rates never exceeded $10 \%$ per 5 minutes, while a large central installation experienced this nearly 2000 times in a one-year period. Additionally, the centralized system experienced ramp rates exceeding 50\% per 5 minutes 17 times and had a peak ramp rate of $65 \%$ per 5 minutes once in the year. These results are consistent with a previous study conducted in the region using pyranometers, supporting the use of measured irradiance data for planning purposes.
\end{abstract}

Keywords-distributed; centralized; photovoltaic; ramp rate; electricity grid; integration

\section{INTRODUCTION}

Substantial decreases in photovoltaic (PV) system prices combined with incentives provided at various levels of government have greatly increased the financial viability of residential installations in many regions around the world. As the number of residential systems continues to grow, it is plausible that the intermittent nature of PV generation will be detrimental to the grid stability. Both predictable (sunrise/set) and transient (cloud cover) phenomena cause rapid fluctuations in the power output from PV systems which in turn can cause electricity grid system voltage and frequency deviations [1].
Fluctuations in PV output can be problematic for utility operators due to the slower response rate of dispatchable generation plants. To mitigate the impact of intermittent generation on the grid, some jurisdictions with significant PV generation have imposed ramp rate restrictions/recommendations (ex. 10\% per 1 minute required by the Puerto Rico Electric Power Authority [2]) to help ensure grid stability [3].

Many researchers use models to assess the intermittent behaviour of PV system fleets. Research by [4] modeled PV smoothing and introduced a metric called dispersion factor which is a function of cloud speed, PV fleet configuration, and the time interval of interest. A limitation of the dispersion factor metric is that it assumes consistent PV system orientation, size, and spacing for the entire fleet; obviously this is not the case in a diverse residential PV system fleet. Modeled data often relies on interpolation methods to take historic insolation data and apply it to a wide geographic area.

Measured insolation values at real sites provide a better representation of real-world installations due to the inclusion of physical system parameters like slope and azimuth, along with localized weather conditions. Research by [5] used a $3 \times 3$ grid of pyranometers to collect data over a $4 \mathrm{x} 4 \mathrm{~km}$ region to investigate smoothing over a region and found that distributing sensors reduced output fluctuations by an average of $40 \%$ compared to single sensor readings. Similar results were obtained by [6], using 6 pyranometers collecting 1 -second global horizontal irradiance data with spacings ranging from 0.69 to $2.47 \mathrm{~km}$. They found that irradiance data were uncorrelated for timescales longer than 10 minutes, showing the importance of shorter timestep data. Research by [7] used measured pyranometer data to model a fleet of 14 large solar plants totalling $20 \mathrm{MW}$ and interplant distances ranging from 10 to $1065 \mathrm{~km}$ and found that there are diminishing returns when increasing the number of plants, and that smoothing had a greater impact at shorter time scales. These works represent radically different spatial scales and do not capture the impacts of intermittent generation spread across a municipality. Research by [8] used pyranometer data from 215 homes in Nova Scotia, Canada to contrast centralized and distributed PV output. Using 1-minute data (down sampled to 5 and 15 minutes) they found that distributed systems had much smaller ramp rates than a centralized group of pyranometers. Additionally, this difference was enhanced when reducing the 
time scale, showing the importance of obtaining high resolution measurements. A drawback of using pyranometer data is that it represents a small point on the roof of a home. This may be insignificant when considering cloud cover but could miss out on important real-world effects such as localised shading of a portion of the roof throughout the day. It also is not able to capture the effect of solar output "clipping" where peak PV output is sacrificed to reduce the cost of inverters. For these reasons, the use of measured PV data is useful for detailed analyses.

To better understand real-system dynamics, researchers use real power production data from installed PV sites. Production data from seven PV plants totalling $20 \mathrm{MW}$ was collected by [9] in 2009 to investigate the effect of system spacing using real world data. One second data for plants separated by 6 to $360 \mathrm{~km}$ was analyzed. The authors found that $6 \mathrm{~km}$ of distance was enough to decorrelate plant outputs, and that the number of plants had a greater effect than geographical spacing on resource smoothing. Research by [10] collected data from 50 PV systems in the Gujarat area of India spaced up to $470 \mathrm{~km}$. They found that most smoothing in the frequency domain occurs in the first 4-5 plants added, with diminishing returns for plants added after this point. An important discussion point addressed by the authors was that results of these studies are specific to a geographic region. This highlights the importance of both geographic conditions and weather patterns on the results of irradiance studies. Research by [11] analyzed power output of 553 homes in a neighbourhood of Ota City, Japan and found that geographic smoothing was more significant for shorter timescales. They also found that above a certain number of homes, additional systems did not reduce system output variability. This study is interesting because it provides insight into the effect of mass residential adoption (80\% of homes in the studied neighbourhood had PV installations). Research by [12] also studied residential installations and found that longer time intervals require greater geographic spacing for smoothing and that spacing can be dependant on the time of year. It was found that shorter spacings were required during the winter months compared to summer months. This difference was attributed to the increased number of completely overcast days in the winter and the low number of perfect clear sky days during the summer.

The above literature has clearly shown potential for ramprate intermittency reduction through diversification of sites, both at residential and commercial scales. However, there is a gap in comparing residential and commercial PV systems at municipal spatial scales using measured power production data, and this is the objective of this study. Of interest is the effect of geographic and temporal smoothing over a municipal scale of hundreds of kilometers. We contrast our findings against those of pyranometer based models to help bridge the gap in model confidence. The results are intended to support future PV policies (e.g. net-metering; self-consumption) and programs (e.g. financial incentives) by providing consideration to power ramp-rate benefits of residential versus commercial systems.

\section{DATA AND METHODS}

This study makes use of AC production values from PV installations located within the Halifax Regional Municipality
(HRM), Nova Scotia, Canada. As part of the Halifax Solar City 2 program the municipality collects inverter data ${ }^{1}$ from installed systems. Currently, only one brand of microinverter (Enphase) is being stored in the database. At the time of writing, there are 99 systems reporting, with a total module count of 2552, and a peak output of $645 \mathrm{~kW}_{\mathrm{ac}}$. Of these, 44 systems $\left(290 \mathrm{~kW}_{\mathrm{ac}}\right)$ were used in this study due to quality control measures discussed in Section II.A. To contrast residential systems against a larger commercial installation, AC production data from a commercial location (IKEA store) located in Dartmouth, Nova Scotia, Canada (part of the HRM) was used. This system uses 28 Fronius string inverters with a total system capacity of $650 \mathrm{~kW}_{\mathrm{ac}}$. This provides an ideal system for comparison with the residential dataset, since the physical system parameters better represent commercial installations compared to using a single home from the residential dataset._Several quality control measures were implemented for this dataset and are addressed in Section II.B. Both datasets have a timestep resolution of 5 minutes.

\section{A. Residential (distributed) data}

Residential data comes from the Halifax Solar City program which provides financing for PV projects within the municipality. Data from a subset of systems, particularly those with micro-inverters, are publicly available. Output data is 5minute average power generation in watts listed for each system, including the number of reporting PV modules. Postal code forward sortation area (FSA) is provided for each site and indicates the geographic location within the municipality.

A total of 99 systems were reporting as of 1 Sep 2019. Only systems which reported for the complete year of 1 Sep 2018 to 1 Sep 2019 were used so that seasonal effects could be identified. 44 systems were used in this study. Raw power data (watts) was normalized for each system based on the maximum reported value within the dataset for that system.

The use of production data encapsulates the physical characteristics of PV systems, and results in a wide array of PV output patterns, as shown in Figure 1. A negative aspect to using measured data is the potential for problems with the data quality, leading to false generation, or a failure to report output. In order to address data quality issues several methods were implemented. First, visual inspection of each system's power vs time of day plot was done to identify systems with very poor data quality. This resulted in the removal of two systems. Further filtering at the system level was done to remove systems which had more than 50 days with missing data, or more than 14 days in a row of missing data. This led to the removal of 14 systems, resulting in a total dataset of 44 systems.

Next, system output values which occurred when less than 10 systems were reporting data were removed. This was done to remove values which occurred early in the morning or late at night. While this likely removes some legitimate data points, the power output of values occurring during these times are very small since all systems are in the same geographical area and so are unlikely to have significant power production during times other systems are offline.

1. Publicly available data from the HRM available at https://catalogue-hrm.opendata.arcgis.com/ 


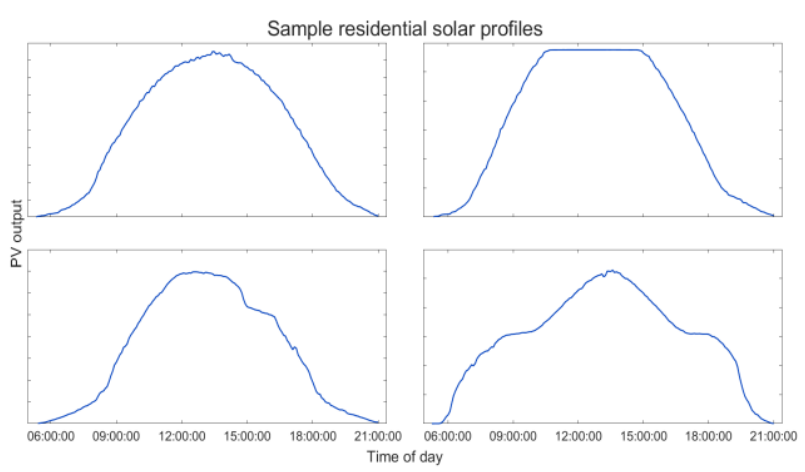

Figure 1. Sample output from four systems vs time on a clear day

\section{B. Commerical (centralized) data}

Commercial data for this study was provided by a large commercial building located centrally within the HRM. This building has a $\sim 650 \mathrm{~kW}_{\mathrm{ac}}$ solar array installed on the roof, with output values shown in Figure 2. The dataset consists of 5minute timestep production data from 28 string inverters. The centralized system is represented by the normalized sum of all 28 reporting inverters. Data recordings for this site began in Nov 2017 and is ongoing. The largest observed power output from the dataset was used to normalize data. This is consistent with how residential data was normalized. There appears to be slight amounts of clipping occurring in the profile due to the lack of a distinct production peak.

As with the residential data, several data quality control steps were implemented: 1) due to interconnection agreements the controls must ensure PV production never exceeds building load causing export to grid. This means that when net load reaches $\sim 50 \mathrm{~kW}$, solar output is curtailed. To avoid including curtailed data, any time the net load fell below $60 \mathrm{~kW}$ corresponding PV data was excluded from the analysis. 2) As with the residential dataset, repeating data values which exceeded realistic power outputs were discovered in the dataset and were deleted. 3) Points which had less than 20 of the 28 inverters reporting were removed from the analysis. Since all the panel strings are in the same geographical location and have the same slope and azimuth, it is very unlikely that 8 of them would be offline when 20 others are online. 4) A filter was implemented which flagged any stretch of three datapoints in a row which had the exact same power value. These data were removed.

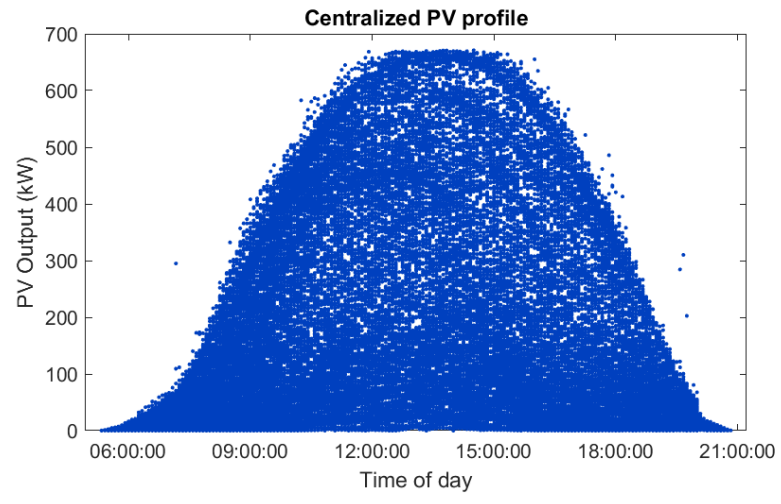

Figure 2. Centralized PV system power output vs time of day

\section{Grid load data}

To examine the influence an increase in PV energy production has on provincial grid operations, grid load data was needed. Provincial net load data for 2018 and 2019 was provided by the provincial electric utility Nova Scotia Power Inc. at a 2-minute timestep. Because it is net load, these data are influenced by the solar output. However, because the load (minimum load $\sim 680 \mathrm{MW}$ ) is so much larger than the PV production (maximum production $<3 \mathrm{MW}$ ), this influence can reasonably be ignored.

\section{RESULTS}

\section{A. Solar production}

A scatterplot of the aggregated residential systems is shown in Figure 3. Interestingly, there is no evidence of clipping based on visual inspection, indicating most of the systems in the dataset do not have a large DC:AC ratio. Clipping is often done to reduce the up-front capital cost of PV systems at the expense of reducing total energy generation and was expected to be more prevalent in the distributed dataset.

Differences in the daily solar profile of each system can be observed by plotting the time-synced system output for specific days of the year. Plots of normalized power generation for both the aggregated residential system and the commercial system for 27-28 Aug 2019 are shown in Figure 4. These dates were used since they represent an intermittent (cloudy) day and a clear day for comparison. During the cloudy day both systems experience many power fluctuations. While the centralized system reaches larger normalized values, it has much sharper rises and falls which would need to be matched by dispatchable generation. The issue of partial shading of a centralized system is shown on the sunny day example. While the distributed system shows a smooth curve typical of solar irradiance the centralized system has a more jagged pattern which is likely caused by cloud cover obscuring part of the array. The centralized array has larger normalized values throughout most of the day. In ideal conditions this is expected because of the uniformity of the centralized system.

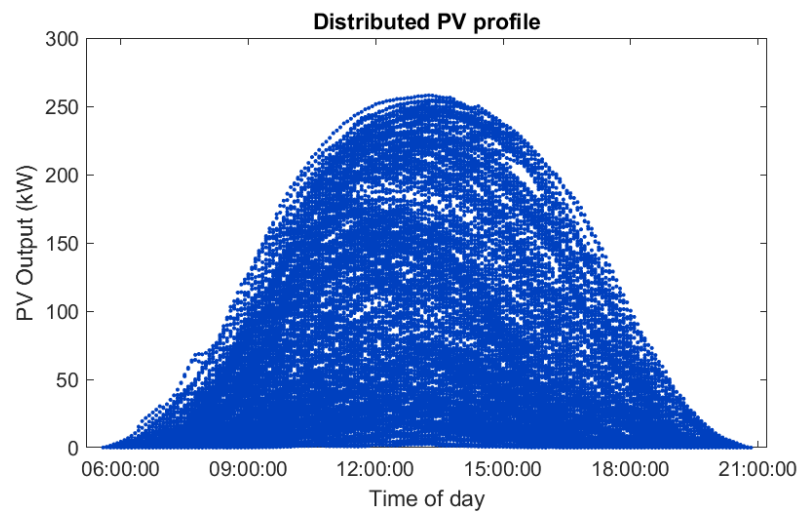

Figure 3. Aggregated residential system profile 


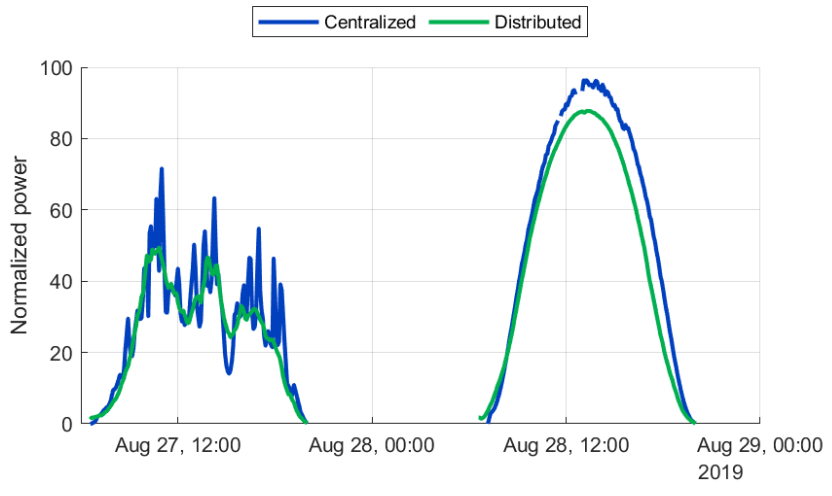

Figure 4. Normalized power for a cloudy and sunny day

\section{B. Ramp rate analysis}

Because data was supplied in 5-minute timesteps, 5-minute ramp rates were obtained by a simple differentiation of the series. 15-minute ramp rates were calculated using the difference between a given value and the timestep 2 values prior. Figure 5. shows the distribution of ramp rate occurrences for each data source when examined on a 5-minute and 15minute timestep.

As expected, small ramp rates dominate the distribution, which requires the use of a $\log$ scale for the number of occurrences. Distributions are mostly symmetrical, indicating sunrise, sunset, and cloud cover/relief rates occur at the same rate. Ramp rates greater than $50 \%$ are not shown. There were 23 occurrences of ramp rates exceeding $50 \%$ per 5 minutes, and 119 exceeding $50 \%$ per 15 minutes for the centralized dataset. There were no occurrences of ramp rates greater than $10 \%$ per 5 minutes for the distributed residential dataset. The maximum ramp rate value observed for the centralized dataset was $65 \%$ per 5 minutes. This greatly exceeds the largest value seen in the distributed dataset (10\% per 5 minutes) and strongly supports previous literature which highlights the benefits of distributing solar generation assets across a given geographical area. Since the exact locations of the residential generators are unknown, an analysis of how distance affects ramp rates in the municipality could not be conducted.

5-minute timestep data was down sampled to 15 -minutes by differentiating each output power value by the value which occurred $\mathrm{n}$-2 timesteps prior. The ramp rate distribution for both datasets was wider when the 5-minute data was down sampled to 15-minute intervals. This is consistent with results obtained by [8]. While at first glance this appears to show that 15-minute timeseries data is a conservative approach, it is important to note that 5-minute ramp rates have a much greater impact on grid stability. The importance of finer resolution timeseries data is dependent on the ability of other generation methods to respond to disruptions.
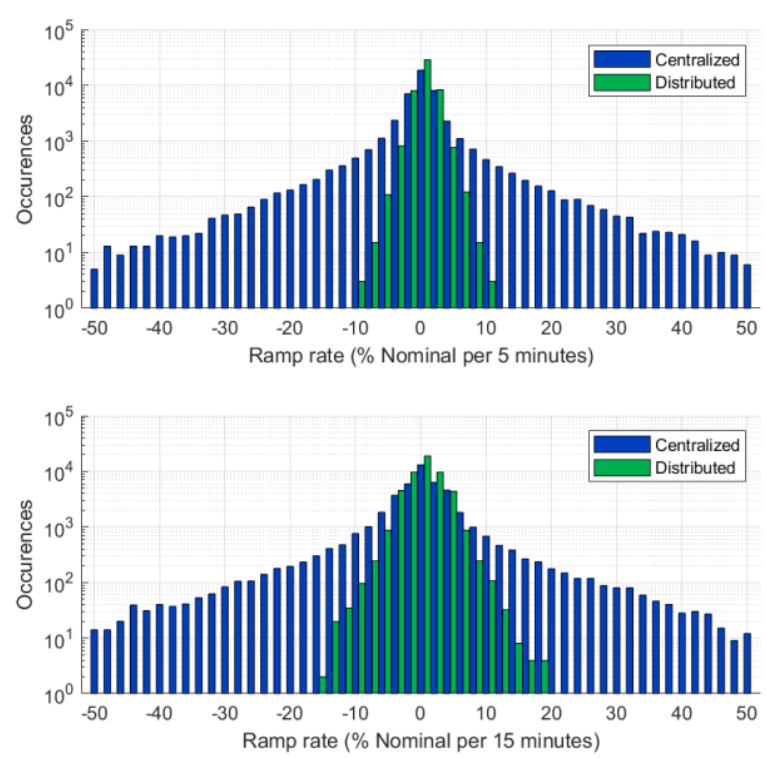

Figure 5. 5 and 15-minute ramp rate occurences

\section{Integration with load}

To examine the interaction between solar resource variability and provincial electrical load, each normalized dataset was scaled up to $100 \mathrm{MW}$ and aligned with load data for Nova Scotia. Two weeks of data were selected (one in the summer and one in the winter) for comparison of how well PV production matches grid loads. These are shown in Figure 6. and Figure 7.

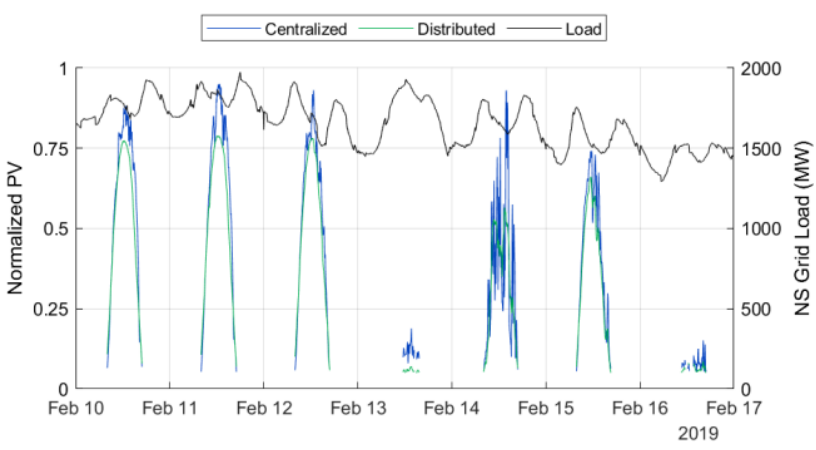

Figure 6. Sample winter week of grid load and normalized PV production

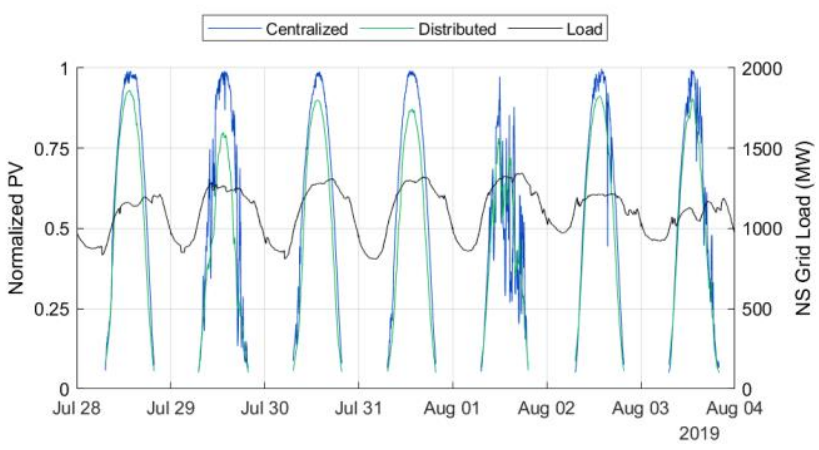

Figure 7. Sample summer week of grid load and normalized PV production 
During the week in February, PV production and load do not align very well. This was expected for the region, as there is a noticeable increase in load during the evening hours while $\mathrm{PV}$ production is decreasing as the sun sets. Interestingly, during days with particularly low PV production (February $13^{\text {th }}$ and $16^{\text {th }}$ ) this effect is less pronounced, particularly on the $13^{\text {th }}$. The weather on this date was cold and had a mix of freezing rain and snow which resulted in the closure of schools in the area. Cold temperatures and substantial cloud cover would increase heating requirements, leading to a high grid load on this day. Typically, during the winter, load increases early in the morning and later in the evening in Nova Scotia, during which time solar output is at its lowest.

Conversely, Figure 7. shows that Nova Scotia summer loads and PV generation align well with a smoother peak load occurring throughout the day. Instead of distinct morning and evening peak loads there is a sharp increase in load in the morning which persists throughout the day before falling late in the evening. The morning ramp aligns well with PV generation rising in the morning, but loads persist much later than PV generation. It should be noted the overall load during the summer is $\sim 500 \mathrm{MW}$ less than during the winter.

\section{CONCLUSION}

Falling PV system prices and favorable incentives have increased the financial viability of residential and commercial PV systems. As more systems come online grid stability may be impacted by the rapid fluctuation in power produced from these intermittent sources. This study used production data recorded between 1 Sep 2018 and 1 Sep 2019. Two sources of data were used to represent different PV systems; an aggregate of installed residential PV systems and one large installation on a commercial building. 15-minute ramp rates had a wider distribution of ramp rate occurrences for both datasets; showing that finer resolution data is important for capturing severe ramp rates. At both timescales, centralized production resulted in much more severe ramp rates. Distributed ramp rates did not exceed $10 \%$ per 5 minutes, while the centralized system experienced up to $65 \%$ per 5 minutes.

Results of this study support measures which would increase distributed generation if more PV is to be allowed to enter the grid. The data suggest targeting the residential market. This needs to be met with some caution however, since smoothing could be observed if there were multiple large commercial datasets available. Given that most large commercial buildings are clustered in a few areas of the municipality, smoothing may not be as significant and warrants further investigation. Large commercial installations may also be offset by increased residential generation, benefitting the municipality as the large installations provide increased energy production while smaller installations in residential areas enhance geographic smoothing effects.

\section{ACKNOWLEDGMENT}

We are grateful to the Nova Scotia Department of Energy and Mines for funding this research through a collaborative agreement with Nova Scotia Community College. We appreciate Halifax Regional Municipality publicly posting high timestep resolution solar production data to its data portal.

\section{REFERENCES}

[1] [1] R. Shah, N. Mithulananthan, R. C. Bansal, and V. K. Ramachandaramurthy, "A review of key power system stability challenges for large-scale PV integration," Renewable and Sustainable Energy Reviews. 2015.

[2] [2] G. Vahan and S. Booth, "Review of PREPA technical requirements for interconnecting wind and solar generation," Natl. Renew. Energy Lab. (NREL), Tech. Rep. NREL/TP-5D00-57089., no. November, p. 72, 2013.

[3] [3] S. Sukumar, M. Marsadek, K. R. Agileswari, and H. Mokhlis, "Ramp-rate control smoothing methods to control output power fluctuations from solar photovoltaic (PV) sources-A review," Journal of Energy Storage, vol. 20. Elsevier Ltd, pp. 218-229, 01-Dec-2018.

[4] [4] T. E. Hoff and R. Perez, "Quantifying PV power Output Variability," Sol. Energy, vol. 84, no. 10, pp. 1782-1793, 2010.

[5] [5] K. Otani, J. Minowa, and K. Kurokawa, "Study on areal solar irradiance for analyzing areally-totalized PV systems," Sol. Energy Mater. Sol. Cells, vol. 47, no. 1-4, pp. 281-288, 1997.

[6] [6] M. Lave, J. Kleissl, and E. Arias-Castro, "High-frequency irradiance fluctuations and geographic smoothing," Solar Energy. 2012.

[7] [7] N. Riaz, S. Repo, and A. V. Lindfors, "Statistical Impact Evaluation of Stochastic Parameters Enhancing Solar Power Inherent Smoothing," 2017 IEEE PES Innovative Smart Grid Technologies Conference Europe, ISGT-Europe 2017 - Proceedings, vol. 2018-Janua. pp. 1-6, 2018.

[8] [8] K. Adye, N. Pearre, and L. Swan, "Contrasting distributed and centralized photovoltaic system performance using regionally distributed pyranometers," Sol. Energy, vol. 160, pp. 1-9, Jan. 2018.

[9] [9] J. Marcos, L. Marroyo, E. Lorenzo, and M. García, "Smoothing of PV power fluctuations by geographical dispersion," Prog. Photovoltaics Res. Appl., vol. 20, no. 2, pp. 226-237, Mar. 2012.

[10] [10] K. Klima and J. Apt, "Geographic smoothing of solar PV: Results from Gujarat," Environ. Res. Lett., vol. 10, no. 10, 2015.

[11] [11] M. Lave, J. S. Stein, A. Ellis, C. W. Hansen, and E. Nakashima, "Ota City: Characterizing Output Variability from 553 Homes with Residential PV Systems on a Distribution Feeder," no. November, 2011.

[12] [12] B. Elsinga and W. van Sark, "Spatial power fluctuation correlations in urban rooftop photovoltaic systems," Prog. Photovoltaics Res. Appl., vol. 23, pp. 1390-1397, 2014. 